\title{
A CLASS OF WEIGHTED CONVOLUTION FRÉCHET ALGEBRAS
}

\author{
THOMAS VILS PEDERSEN \\ Department of Basic Sciences and Environment, University of Copenhagen \\ Thorvaldsensvej 40, DK-1871 Frederiksberg C, Denmark \\ E-mail:vils@life.ku.dk
}

\begin{abstract}
For an increasing sequence $\left(\omega_{n}\right)$ of algebra weights on $\mathbb{R}^{+}$we study various properties of the Fréchet algebra $A(\omega)=\bigcap_{n} L^{1}\left(\omega_{n}\right)$ obtained as the intersection of the weighted Banach algebras $L^{1}\left(\omega_{n}\right)$. We show that every endomorphism of $A(\omega)$ is standard, if for all $n \in \mathbb{N}$ there exists $m \in \mathbb{N}$ such that $\omega_{m}(t) / \omega_{n}(t) \rightarrow \infty$ as $t \rightarrow \infty$. Moreover, we characterise the continuous derivations on this algebra: Let $M\left(\omega_{n}\right)$ be the corresponding weighted measure algebras and let $B(\omega)=\bigcap_{n} M\left(\omega_{n}\right)$. If for all $n \in \mathbb{N}$ there exists $m \in \mathbb{N}$ such that $t \omega_{n}(t) / \omega_{m}(t)$ is bounded on $\mathbb{R}^{+}$, then the continuous derivations on $A(\omega)$ are exactly the linear maps $D$ of the form $D(f)=(X f) * \mu$ for $f \in A(\omega)$, where $\mu \in B(\omega)$ and $(X f)(t)=t f(t)$ for $t \in \mathbb{R}^{+}$and $f \in A(\omega)$. If the condition is not satisfied, we show that $A(\omega)$ has no non-zero derivations.
\end{abstract}

1. Introduction. In this paper we will study a class of Fréchet algebras, which are intersections (or more formally projective limits) of decreasing sequences of weighted convolution Banach algebras on the half-line. We start by recalling the definition and some basic properties of these algebras (see for instance 16 for further details).

Let $L^{1}\left(\mathbb{R}^{+}\right)$be the Banach space of (equivalence classes of) integrable functions $f$ on $\mathbb{R}^{+}=[0, \infty)$ with the norm $\|f\|=\int_{0}^{\infty}|f(t)| d t$. A positive Borel function $\omega$ on $\mathbb{R}^{+}$ is called an algebra weight if (a) $\omega$ and $1 / \omega$ are locally bounded on $\mathbb{R}^{+}$, (b) $\omega$ is right continuous on $\mathbb{R}^{+}$, (c) $\omega$ is submultiplicative, that is $\omega(t+s) \leq \omega(t) \omega(s)$ for $t, s \in \mathbb{R}^{+}$, and $(\mathrm{d}) \omega(0)=1$. We then define $L^{1}(\omega)$ as the weighted space of functions $f$ on $\mathbb{R}^{+}$for which $f \omega \in L^{1}\left(\mathbb{R}^{+}\right)$with the inherited norm

$$
\|f\|_{\omega}=\int_{0}^{\infty}|f(t)| \omega(t) d t .
$$

2010 Mathematics Subject Classification: Primary 46J99, 46E30, 47B47.

Key words and phrases: Fréchet algebras, weighted convolution algebras, endomorphisms, derivations.

The paper is in final form and no version of it will be published elsewhere. 
With the usual convolution product

$$
(f * g)(t)=\int_{0}^{t} f(s) g(t-s) d s \quad \text { for } t \in \mathbb{R}^{+} \text {and } f, g \in L^{1}(\omega)
$$

it is well known that $L^{1}(\omega)$ is a commutative Banach algebra. Similarly, the space $M(\omega)$ of locally finite complex Borel measures $\mu$ on $\mathbb{R}^{+}$for which

$$
\|\mu\|_{\omega}=\int_{0}^{\infty} \omega(t) d|\mu| t<\infty
$$

is a Banach algebra under convolution and contains $L^{1}(\omega)$ as a closed ideal.

We are now ready to define the Fréchet algebras which we will study. Throughout this paper $\omega=\left(\omega_{n}\right)$ will denote an increasing sequence of algebra weights on $\mathbb{R}^{+}$and we let

$$
A(\omega)=\bigcap_{n} L^{1}\left(\omega_{n}\right) \quad \text { and } \quad B(\omega)=\bigcap_{n} M\left(\omega_{n}\right) .
$$

Equipped with the increasing sequence of norms $\|\mu\|_{n}=\|\mu\|_{\omega_{n}}(\mu \in B(\omega))$, it is easily seen that $A(\omega)$ and $B(\omega)$ become Fréchet algebras. More abstractly, these Fréchet algebras can be viewed as projective limits of the weighted Banach algebras in question (with the inclusion maps). In particular, sets of the form $\left\{g \in A(\omega):\|g\|_{L^{1}\left(\omega_{m}\right)}<\delta\right\}$ with $\delta>0$ and $m \in \mathbb{N}$ form a base at zero for the projective limit topology on $A(\omega)$. For a sequence $\left(f_{k}\right)$ in $A(\omega)$ we thus have $f_{k} \rightarrow 0$ in $A(\omega)$ as $k \rightarrow \infty$ if and only if $f_{k} \rightarrow 0$ in $L^{1}\left(\omega_{n}\right)$ as $k \rightarrow \infty$ for every $n \in \mathbb{N}$. We refer to Michael's original memoir [21] for general background material on Fréchet algebras.

In the rest of the paper we will make the following assumptions on the weights $\omega=$ $\left(\omega_{n}\right)$ :

(a) $\omega_{n}(t) \rightarrow \infty$ as $t \rightarrow \infty$ for every $n \in \mathbb{N}$,

(b) $\lim _{t \rightarrow \infty} \omega_{n}(t)^{1 / t}=1$ for every $n \in \mathbb{N}$,

(c) $\sup _{t \in \mathbb{R}^{+}} \omega_{n+1}(t) / \omega_{n}(t)=\infty$ for every $n \in \mathbb{N}$.

It follows from (a) that the weights are semisimple, and the assumption (b) is equivalent to each of the algebras $L^{1}\left(\omega_{n}\right)$ having the right hand half-plane as character space (see the proof of Theorem 2.3). Moreover, condition (c) means that $L^{1}\left(\omega_{n+1}\right) \subset L^{1}\left(\omega_{n}\right)$, so that the descending chain $\left(L^{1}\left(\omega_{n}\right)\right)$ does not stabilise. We are mainly interested in the case where we further have

(d) $\omega_{n}(t) \rightarrow \infty$ as $n \rightarrow \infty$ for every $t \in \mathbb{R}^{+}$,

but we will see that there are also interesting aspects in the case where the "limit weight" $\omega_{\infty}(t)=\lim _{n \rightarrow \infty} \omega_{n}(t)$ is finite (for instance $\omega_{n}(t)=(1+t)^{1-1 / n}$ for $t \in \mathbb{R}^{+}$and $n \in \mathbb{N}$ ). It may also be of interest to study $A(\omega)$ for radical weights $\left(\omega_{n}\right)$ with $\omega_{\infty}$ either radical or semisimple.

The organisation of the paper is as follows: In Section 2 we collect various basic results about the algebra $A(\omega)$ including a characterisation of $B(\omega)$ as the multiplier algebra of $A(\omega)$. Endomorphisms of $A(\omega)$ are studied in Section 3. In particular, under a slightly stronger assumption than (c) above we show that every endomorphism of $A(\omega)$ is standard. Finally, in Section 4 we describe the derivations on $A(\omega)$. 
2. Basic results. In this section we present some basic results about the algebras $A(\omega)$ and $B(\omega)$. The following result is a direct consequence of the topology on $A(\omega)$.

Lemma 2.1. A linear map $T: A(\omega) \rightarrow A(\omega)$ is continuous if and only if for all $n \in$ $\mathbb{N}$ there exists $m \in \mathbb{N}$ such that $T$ extends continuously to a map (also denoted) $T$ : $L^{1}\left(\omega_{m}\right) \rightarrow L^{1}\left(\omega_{n}\right)$

Similarly, a linear functional $\varphi: A(\omega) \rightarrow \mathbb{C}$ is continuous if and only if $\varphi$ extends continuously to $L^{1}\left(\omega_{n}\right)$ for some $n \in \mathbb{N}$.

Proof. Clearly $T: A(\omega) \rightarrow A(\omega)$ is continuous if the continuous extensions exist. Conversely, assume that $T: A(\omega) \rightarrow A(\omega)$ is continuous and let $n \in \mathbb{N}$. Since $U=\{f \in$ $\left.A(\omega):\|f\|_{n}<1\right\}$ is an open neighbourhood of 0 in $A(\omega)$, the same holds for $T^{-1}(U)$, so there exist $\delta>0$ and $m \in \mathbb{N}$ such that $\left\{g \in A(\omega):\|g\|_{L^{1}\left(\omega_{m}\right)}<\delta\right\} \subseteq T^{-1}(U)$. Hence $\|T g\|_{n} \leq \frac{1}{\delta}\|g\|_{m}$ for $g \in A(\omega)$, so a standard argument using Cauchy sequences shows that $T$ extends continuously to a map $T: L^{1}\left(\omega_{m}\right) \rightarrow L^{1}\left(\omega_{n}\right)$. Similarly for the second part of the lemma.

For a weight $\omega$ let $L^{\infty}(1 / \omega)$ denote the Banach space of functions $h$ on $\mathbb{R}^{+}$for which $\|h\|=\sup _{t \in \mathbb{R}^{+}}|h(t)| / \omega(t)<\infty$. It is well known that the duality $\langle f, h\rangle=\int_{0}^{\infty} f(t) h(t) d t$ for $f \in L^{1}(\omega)$ and $h \in L^{\infty}(1 / \omega)$ identifies $L^{\infty}(1 / \omega)$ isometrically isomorphically with the dual space of $L^{1}(\omega)$. As a consequence of the previous lemma we therefore have the following characterisation of the dual space of $A(\omega)$.

COROLlary 2.2. Every function $h \in \bigcup_{n} L^{\infty}\left(1 / \omega_{n}\right)$ defines a continuous linear functional on $A(\omega)$ by $\langle f, h\rangle=\int_{0}^{\infty} f(t) h(t) d t(f \in A(\omega))$ and conversely every continuous linear functional on $A(\omega)$ is of this form.

TheOREM 2.3. Every character on $A(\omega)$ is continuous. Moreover, for every $z \in \mathbb{C}$ with Re $z \geq 0$ the Laplace transform

$$
\mathcal{L}(f)(z)=\int_{0}^{\infty} f(t) e^{-z t} d t \quad(f \in A(\omega))
$$

defines a continuous character on $A(\omega)$ and conversely every character on $A(\omega)$ has this form.

Proof. For $z \in \mathbb{C}$ with $\operatorname{Re} z \geq 0$ the map $f \mapsto \mathcal{L}(f)(z)(f \in A(\omega))$ defines a continuous character on each $L^{1}\left(\omega_{n}\right)$ by the usual characterisation of the characters on $L^{1}\left(\omega_{n}\right)$ (4 , Theorem 4.7.27]) and hence defines a continuous character on $A(\omega)$. Let $g(t)=e^{-t}$ $(t \geq 0)$. It follows from [4, Theorem 4.7.26] that the constant function with value 1 is a polynomial generator of $L^{1}\left(e^{-t} \omega_{n}(t)\right)$ and thus that $g$ is a polynomial generator of $L^{1}\left(\omega_{n}\right)$ for every $n \in \mathbb{N}$. Hence $g$ is a polynomial generator of $A(\omega)$, so every character on $A(\omega)$ is continuous by [4, Corollary 4.10.11]. By Lemma 2.1 it thus follows that every character $\varphi: A(\omega) \rightarrow \mathbb{C}$ extends continuously to $L^{1}\left(\omega_{n}\right)$ for some $n \in \mathbb{N}$. Hence there exists $z \in \mathbb{C}$ with $\operatorname{Re} z \geq 0$ such that $\varphi(f)=\mathcal{L}(f)(z)$ for $f \in L^{1}\left(\omega_{n}\right)$.

It follows from Theorem 2.3 that the maximal modular ideals in $A(\omega)$ are exactly the sets $\{f \in A(\omega): \mathcal{L}(f)(z)=0\}$, where $z \in \mathbb{C}$ with $\operatorname{Re} z \geq 0$. The corresponding sets $\{\mu \in B(\omega): \mathcal{L}(\mu)(z)=0\}$ are maximal ideals in $B(\omega)$ and their intersection equals $\{0\}$, so we have the following result. 
COROLlary 2.4. The Fréchet algebras $A(\omega)$ and $B(\omega)$ are semisimple.

It is well known that each $L^{1}\left(\omega_{n}\right)(n \in \mathbb{N})$ has a bounded approximate identity, for instance $e_{k}(t)=k \cdot 1_{[0,1 / k]}(k \in \mathbb{N})$. Hence $e_{k} * f \rightarrow f$ in $A(\omega)$ as $k \rightarrow \infty$ for $f \in A(\omega)$, so $\left(e_{k}\right)$ is also a bounded approximate identity for $A(\omega)$. However, if $\lim _{n \rightarrow \infty} \omega_{n}(t)=\infty$ for every $t \in \mathbb{R}^{+}$(which is the case we are most interested in), then $\|f\|_{n} \rightarrow \infty$ as $n \rightarrow \infty$ for every non-zero $f \in A(\omega)$. Hence $A(\omega)$ does not possess a uniformly bounded approximate identity, that is, a bounded approximate identity where the bound in $L^{1}\left(\omega_{n}\right)$ is independent of $n \in \mathbb{N}$. Note, however, that $\left\|e_{k}\right\|_{n} \rightarrow 1$ as $k \rightarrow \infty$ for every $n \in \mathbb{N}$.

For a Fréchet algebra resp. Fréchet module with a uniformly bounded approximate identity, Craw ([3]) resp. Summers ([24]) generalised Cohen's factorisation theorem. (See also 7] for a thorough discussion of approximate identities and factorisation.) These results do not apply to $A(\omega)$ if $\lim _{n \rightarrow \infty} \omega_{n}(t)=\infty$ for every $t \in \mathbb{R}^{+}$and we do not know whether we have factorisation in $A(\omega)$ or in Fréchet $A(\omega)$-modules; not even in the simplest sense of being able to factor every $f \in A(\omega)$ as a product $f=g * h$ with $g, h \in A(\omega)$. We complement the discussion by mentioning that in the special case where there exists $a>0$ such that $\sup _{n \in \mathbb{N}, 0 \leq t \leq a} \omega_{n}(t)<\infty$, the sequence $\left(e_{k}\right)$ is a uniformly bounded approximate identity for $A(\omega)$, and we therefore have factorisation in $A(\omega)$.

A linear map $T$ on a commutative Fréchet algebra $\mathcal{B}$ is called a multiplier if $T(a b)=$ $T(a) b$ for every $a, b \in \mathcal{B}$. Recall from [8, Theorem 2.2] that every $\mu \in M(\omega)$ defines a continuous multiplier $T_{\mu}$ on $L^{1}(\omega)$ by $T_{\mu}(f)=\mu * f\left(f \in L^{1}(\omega)\right)$ and that this identifies $M(\omega)$ isometrically isomorphically with the multiplier algebra of $L^{1}(\omega)$. For the algebra $A(\omega)$ we have a similar result.

Theorem 2.5. Every $\mu \in B(\omega)$ defines a continuous multiplier $T_{\mu}$ on $A(\omega)$ by $T_{\mu}(f)=$ $\mu * f(f \in A(\omega))$ and every multiplier on $A(\omega)$ has this form. In particular, every multiplier on $A(\omega)$ is continuous.

Proof. Clearly $T_{\mu}$ is a continuous multiplier on $A(\omega)$ for every $\mu \in B(\omega)$. Conversely, let $T$ be a multiplier on $A(\omega)$. Since $A(\omega)$ is an integral domain, it is well known (see [4. Proposition 2.5.12]) that $T$ is automatically continuous. Let $\left(e_{k}\right)$ be a bounded approximate identity for $A(\omega)$ and let $n \in \mathbb{N}$. By Lemma 2.1 there exists $m \in \mathbb{N}$ such that $T$ extends continuously to a map $T: L^{1}\left(\omega_{m}\right) \rightarrow L^{1}\left(\omega_{n}\right)$. Hence the sequence $\left(T e_{k}\right)$ is bounded in $L^{1}\left(\omega_{n}\right)$, and it follows from the proof of [4, Theorem 3.3.40] that the sequence has a weak-star cluster point $\mu_{n} \in M\left(\omega_{n}\right)$ for which $T(f)=\mu_{n} * f$ for $f \in L^{1}\left(\omega_{n}\right)$. By uniqueness of $\mu_{n}$ it is independent of $n$ and the result follows.

The identification in the previous theorem of $B(\omega)$ as the multiplier algebra of $A(\omega)$ induces a strong operator topology (denoted by SO) on $B(\omega)$ in which a net $\left(\mu_{\beta}\right)$ tends to 0 if and only if $\mu_{\beta} * f \rightarrow 0$ for every $f \in A(\omega)$. We will now introduce another topology on $B(\omega)$, denoted by $\sigma$, which is the projective limit topology, when each of the spaces $M\left(\omega_{n}\right)$ is regarded as the multiplier algebra of $L^{1}\left(\omega_{n}\right)$ and is equipped with the corresponding strong operator topology (denoted by $\mathrm{SO}_{n}$ ). Hence $\sigma$ is the weakest topology on $B(\omega)$ making all the inclusions $\iota_{n}:(B(\omega), \sigma) \rightarrow\left(M\left(\omega_{n}\right), \mathrm{SO}_{n}\right)$ for $n \in \mathbb{N}$ continuous (see, for instance, [21, Proof of Proposition 2.7]). Also, for a net $\left(\mu_{\beta}\right)$ in $B(\omega)$ we have $\mu_{\beta} \rightarrow 0$ in the $\sigma$-topology if and only if $\mu_{\beta} \rightarrow 0$ strongly in $M\left(\omega_{n}\right)$ for all $n \in \mathbb{N}$. 
We will need the following results about the $\sigma$-topology.

LEMma 2.6. We have $S O \subseteq \sigma$, that is, $S O$ is weaker than $\sigma$.

Proof. Let $\left(\mu_{\beta}\right)$ be a net in $B(\omega)$ with $\mu_{\beta} \rightarrow 0$ in the $\sigma$-topology, that is, $\mu_{\beta} \rightarrow 0$ strongly in $M\left(\omega_{n}\right)$ for every $n \in \mathbb{N}$. For $f \in A(\omega)$ we thus have $\mu_{\beta} * f \rightarrow 0$ in $L^{1}\left(\omega_{n}\right)$ for every $n \in \mathbb{N}$ and thus $\mu_{\beta} * f \rightarrow 0$ in $A(\omega)$. Hence $\mu_{\beta} \rightarrow 0$ strongly in $B(\omega)$, so the identity map $\iota:(B(\omega), \sigma) \rightarrow(B(\omega), \mathrm{SO})$ is continuous and the result follows.

We do not know whether the $\sigma$ - and the SO-topologies are identical, but on bounded sets in $B(\omega)$ (that is, bounded in every $M\left(\omega_{n}\right)$ ) that is the case: Let $\left(\mu_{\beta}\right)$ be a bounded net in $B(\omega)$ with $\mu_{\beta} \rightarrow 0$ strongly and let $n \in \mathbb{N}$. Then $\mu_{\beta} * f \rightarrow 0$ in $L^{1}\left(\omega_{n}\right)$ for every $f \in A(\omega)$ and by the boundeness thus for $f \in L^{1}\left(\omega_{n}\right)$. Hence $\mu_{\beta} \rightarrow 0$ strongly in $M\left(\omega_{n}\right)$ for every $n \in \mathbb{N}$ and thus $\mu_{\beta} \rightarrow 0$ in the $\sigma$-topology. Moreover, we have the following result.

Proposition 2.7. The dual spaces of $(B(\omega), \sigma)$ and $(B(\omega), S O)$ are the same, that is, a linear functional on $B(\omega)$ is $\sigma$-continuous if and only if it is strongly continuous.

Proof. It follows from Lemma 2.6 that a strongly continuous linear functional on $B(\omega)$ also is $\sigma$-continuous. Conversely, let $\varphi: B(\omega) \rightarrow \mathbb{C}$ be a $\sigma$-continuous linear functional. Since $\sigma$ is the weakest topology on $B(\omega)$ making all the inclusions $\iota_{n}:(B(\omega), \sigma) \rightarrow$ $\left(M\left(\omega_{n}\right), \mathrm{SO}_{n}\right)$ for $n \in \mathbb{N}$ continuous, it follows that the family of seminorms

$$
p_{n, f}(\mu)=\|\mu * f\|_{n} \quad(\mu \in B(\omega))
$$

with $n \in \mathbb{N}$ and $f \in L^{1}\left(\omega_{n}\right)$ defines the $\sigma$-topology. Hence (see, for instance, [2, Theorem IV.3.1]) there exist $n_{1}, \ldots, n_{J}$ and $f_{j} \in L^{1}\left(\omega_{n_{j}}\right)(j=1, \ldots, J)$ such that

$$
\|\varphi(\mu)\| \leq \sum_{j=1}^{J}\left\|\mu * f_{j}\right\|_{n_{j}} \leq \sum_{j=1}^{J}\left\|\mu * f_{j}\right\|_{N} \quad(\mu \in B(\omega))
$$

with $N=\max \left\{n_{1}, \ldots, n_{J}\right\}$. A standard argument using Cauchy sequences now shows that $\varphi$ extends to a linear functional $\varphi_{N}: M\left(\omega_{N}\right) \rightarrow \mathbb{C}$ with $\left\|\varphi_{N}(\mu)\right\| \leq \sum_{j=1}^{J}\left\|\mu * f_{j}\right\|_{N}$ for $\mu \in M\left(\omega_{N}\right)$. In particular this shows that $\varphi$ is strongly continuous on $B(\omega)$.

The previous proposition does not in itself imply that the $\sigma$ - and the SO-topologies are identical. For instance, if $X$ is a Banach space, then the dual spaces of $X$ with respect to the norm and the weak topologies are identical ([23, p. 63]), whereas the norm and the weak topologies themselves are not.

3. Endomorphisms. Homomorphisms between weighted convolution Banach algebras have been studied extensively; see for instance [16], [8], [14] and [10. For endomorphisms of $A(\omega)$ we start with the following consequence of Theorem 2.3

COROLlary 3.1. Every endomorphism of $A(\omega)$ is continuous. More generally, if $\mathcal{B}$ is a Fréchet algebra on which every character is continuous, then every homomorphism $\Phi: \mathcal{B} \rightarrow A(\omega)$ is continuous. 
Proof. This follows from Theorem 2.3 as in the proof of [4, Theorem 2.3.3] since $A(\omega)$ is semisimple (Corollary 2.4 and since the closed graph theorem holds for operators on Fréchet spaces (4, Theorem A.3.25]).

REmark. It follows fom the proof of [9, Theorem 1] (see also [16, Appendix]) that every endomorphism of $A(\omega)$ is a monomorphism.

The next result (which can be generalised to homomorphisms from $A(\omega)$ to $A(\widetilde{\omega})$ ) is similar to and follows rather easily from the corresponding result for $L^{1}(\omega)$ ([16. Theorems 3.4 and 3.6]).

TheOREM 3.2. Let $\Phi$ be a non-zero endomorphism of $A(\omega)$. Then $\Phi$ has a unique extension to a continuous endomorphism $\widetilde{\Phi}$ of $B(\omega)$. Also, $\nu^{t}=\widetilde{\Phi}\left(\delta_{t}\right)\left(t \in \mathbb{R}^{+}\right)$defines a semigroup in $B(\omega)$ which is strongly continuous for $t>0$. Moreover,

$$
\widetilde{\Phi}(\mu)=\int_{0}^{\infty} \nu^{t} d \mu(t) \quad \text { for } \mu \in B(\omega),
$$

where the integrals exist as strong Bochner integrals in $B(\omega)$, that is, $\widetilde{\Phi}(\mu) * f=\int_{0}^{\infty} \nu^{t} *$ $f d \mu(t)$ exists as a Bochner integral in each $L^{1}\left(\omega_{n}\right)(n \in \mathbb{N})$ for $\mu \in B(\omega)$ and $f \in A(\omega)$.

Proof. By the previous theorem $\Phi$ is continuous. For $n \in \mathbb{N}$ it thus follows from Lemma 2.1 that there exists $m \in \mathbb{N}$ such that $\Phi$ extends continuously and uniquely to a homomorphism $\Phi_{n}: L^{1}\left(\omega_{m}\right) \rightarrow L^{1}\left(\omega_{n}\right)$. By [16, Theorems 3.4 and 3.6], $\Phi_{n}$ extends uniquely to a continuous homomorphism $\widetilde{\Phi}_{n}: M\left(\omega_{m}\right) \rightarrow M\left(\omega_{n}\right)$, the semigroup $\left(\nu^{t}\right)$ is strongly continuous in $L^{1}\left(\omega_{n}\right)$ for $t>0$ and $\widetilde{\Phi}_{n}(\mu) * f=\int_{0}^{\infty} \nu^{t} * f d \mu(t)$ exists as a Bochner integral in $L^{1}\left(\omega_{n}\right)$ for $\mu \in M\left(\omega_{m}\right)$ and $f \in L^{1}\left(\omega_{n}\right)$. By uniqueness $\widetilde{\Phi}(\mu)=\widetilde{\Phi}_{n}(\mu)$ is independent of $n \in \mathbb{N}$ for $\mu \in B(\omega)$, and the result follows.

In the rest of this section we will investigate other continuity properties of the endomorphism $\widetilde{\Phi}$ of $B(\omega)$; namely with respect to a $\mathrm{wk}^{*}$ topology and the strong topology on $B(\omega)$. If we denote by $C_{0}(1 / \omega)$ the closed subspace of $L^{\infty}(1 / \omega)$ consisting of continuous functions $h$ on $\mathbb{R}^{+}$for which $h / \omega$ vanishes at infinity, then it is well known that $M(\omega)$ is isometrically isomorphic to the dual space of $C_{0}(1 / \omega)$ with the duality being defined by

$$
\langle h, \mu\rangle=\int_{0}^{\infty} h(t) d \mu(t) \quad\left(h \in C_{0}(1 / \omega), \mu \in M(\omega)\right) .
$$

We can use this to show that $B(\omega)$ is also a dual space. Let

$$
D(1 / \omega)=\bigcup_{n \in \mathbb{N}} C_{0}\left(1 / \omega_{n}\right)
$$

and equip $D(1 / \omega)$ with the inductive limit topology (see, for instance, [2, Chapter IV.5]). Since a linear functional on $D(1 / \omega)$ is continuous if and only if its restriction to each $C_{0}\left(1 / \omega_{n}\right)$ is continuous (see, for instance, [2, Proposition IV.5.7]) we obtain the following result.

Proposition 3.3. The duality

$$
\langle h, \mu\rangle=\int_{0}^{\infty} h(t) d \mu(t) \quad(h \in D(1 / \omega), \mu \in B(\omega))
$$


identifies $B(\omega)$ with the dual space of $D(1 / \omega)$. Moreover, for a net $\left(\mu_{\beta}\right)$ in $B(\omega)$ we have $\mu_{\beta} \rightarrow 0 w k^{*}$ in $B(\omega)$ if and only if $\mu_{\beta} \rightarrow 0 w k^{*}$ in $M\left(\omega_{n}\right)$ for every $n \in \mathbb{N}$.

For a homomorphism $\Phi: L^{1}\left(\omega_{1}\right) \rightarrow L^{1}\left(\omega_{2}\right)$ it was proved by Grabiner (17, Theorem 1.1]) that the extension $\widetilde{\Phi}: M\left(\omega_{1}\right) \rightarrow M\left(\omega_{2}\right)$ is automatically wk* continuous. (See 22 for related results about homomorphisms from $L^{1}(\omega)$ into other Banach algebras.) The corresponding result for endomorphisms of $A(\omega)$ is an almost direct consequence of Grabiner's result.

THEOREM 3.4. Let $\Phi$ be an endomorphism of $A(\omega)$, let $\widetilde{\Phi}$ be the unique extension to an endomorphism of $B(\omega)$ and let $\left(\nu^{t}\right)$ be the semigroup in $B(\omega)$ given by Theorem 3.2. Then $\widetilde{\Phi}$ is wk* continuous. Moreover, $\left(\nu^{t}\right)$ is $w k^{*}$ continuous in $B(\omega)$ for $t \geq 0$ and for every $n \in \mathbb{N}$ there exists $m \in \mathbb{N}$ such that $\nu^{t} \omega_{n}(t) / \omega_{m}(t) \rightarrow 0 w k^{*}$ in $M\left(\mathbb{R}^{+}\right)$as $t \rightarrow \infty$.

Proof. Let $\left(\mu_{\beta}\right)$ be a net in $B(\omega)$ with $\mu_{\beta} \rightarrow 0 \mathrm{wk}^{*}$ in $B(\omega)$ and let $n \in \mathbb{N}$. By Proposition 3.3 we have $\mu_{\beta} \rightarrow 0 \mathrm{wk}^{*}$ in $M\left(\omega_{n}\right)$. It follows from the proof of Theorem 3.2 that there exists $m \in \mathbb{N}$ such that $\widetilde{\Phi}$ extends to a continuous homomorphism $\widetilde{\Phi}_{n}: M\left(\omega_{m}\right) \rightarrow M\left(\omega_{n}\right)$. By Grabiner's result $\widetilde{\Phi}_{n}$ is wk* continuous, so $\widetilde{\Phi}\left(\mu_{\beta}\right)=\widetilde{\Phi}_{n}\left(\mu_{\beta}\right) \rightarrow 0 \mathrm{wk}^{*}$ in $M\left(\omega_{n}\right)$. Hence $\widetilde{\Phi}\left(\mu_{\beta}\right) \rightarrow 0 \mathrm{wk}^{*}$ in $B(\omega)$, so we deduce that $\widetilde{\Phi}$ is $\mathrm{wk}^{*}$ continuous. Since $\delta_{t}$ is wk* continuous in $B(\omega)$ for $t \geq 0$, it follows that $\nu^{t}=\widetilde{\Phi}\left(\delta_{t}\right)$ is wk* continuous in $B(\omega)$ for $t \geq 0$. Similarly $\delta_{t} / \omega_{m}(t) \rightarrow 0 \mathrm{wk}^{*}$ in $M\left(\omega_{m}\right)$ as $t \rightarrow \infty$, so $\nu^{t} / \omega_{m}(t)=\widetilde{\Phi}\left(\delta_{t} / \omega_{m}(t)\right) \rightarrow 0 \mathrm{wk}^{*}$ in $M\left(\omega_{n}\right)$, that is, $\nu^{t} \omega_{n}(t) / \omega_{m}(t) \rightarrow 0 \mathrm{wk}^{*}$ in $M\left(\mathbb{R}^{+}\right)$as $t \rightarrow \infty$.

For homomorphisms $\Phi: L^{1}\left(\omega_{1}\right) \rightarrow L^{1}\left(\omega_{2}\right)$ it is not known whether the semigroup $\left(\nu^{t}\right)$ is strongly continuous in $M\left(\omega_{2}\right)$ at $t=0$. In the papers [10] and 11 this problem was linked to the notion of convergence factors. Using the $\mathrm{wk}^{*}$ continuity of $\left(\nu^{t}\right)$ at $t=0$ and a result from [11] we can show that under a rather mild growth condition on the weights $\omega_{n}$ as $n \rightarrow \infty$, the semigroup $\left(\nu^{t}\right)$ from Theorem 3.2 is strongly continuous in $B(\omega)$ at $t=0$.

THEOREM 3.5. Let $\Phi$ be a non-zero endomorphism of $A(\omega)$, and let $\left(\nu^{t}\right)$ be the semigroup from Theorem 3.2. Suppose that

$$
\text { for every } n \in \mathbb{N} \text { there exists } m \in \mathbb{N} \text { such that } \frac{\omega_{m}(s)}{\omega_{n}(s)} \rightarrow \infty \text { as } s \rightarrow \infty \text {. }
$$

Then $\left(\nu^{t}\right)$ is strongly continuous in $B(\omega)$ for $t \geq 0$.

Proof. By Theorem 3.2 we only need to prove the strong continuity at $t=0$. Let $n \in \mathbb{N}$ and choose $m \in \mathbb{N}$ such that $\omega_{n}(s) / \omega_{m}(s) \rightarrow 0$ as $s \rightarrow \infty$. Let $\eta=\omega_{n} / \omega_{m}$. Then $\eta$ is bounded and $M\left(\omega_{m} \eta\right)=M\left(\omega_{n}\right)$ is translation invariant. Moreover,

$$
\frac{\omega_{m}(r+s)}{\omega_{m}(s)} \eta(r+s)=\frac{\omega_{n}(r+s)}{\omega_{m}(s)} \leq \omega_{n}(r) \frac{\omega_{n}(s)}{\omega_{m}(s)} \rightarrow 0 \quad \text { as } s \rightarrow \infty
$$

for every $r>0$. It thus follows from [11, Theorem 3.2] that $\eta$ is a convergence factor for $\omega_{m}$ at 0 . This means that if $\left(\mu_{k}\right)$ is a sequence in $M\left(\omega_{m}\right)$ with $\mu_{k} \rightarrow 0 \mathrm{wk}^{*}$ in $M\left(\omega_{m}\right)$ as $k \rightarrow \infty$, then $\mu_{k} * f \rightarrow 0$ in the norm of $L^{1}\left(\omega_{m} \eta\right)=L^{1}\left(\omega_{n}\right)$ as $k \rightarrow \infty$ for every $f \in L^{1}\left(\omega_{n}\right)$, that is, $\mu_{k} \rightarrow 0$ strongly in $M\left(\omega_{n}\right)$ as $k \rightarrow \infty$. By Theorem 3.4 we have $\nu^{t} \rightarrow \widetilde{\Phi}\left(\delta_{0}\right)=\delta_{0} \mathrm{wk}^{*}$ in $M\left(\omega_{m}\right)$ as $t \rightarrow 0$, so we conclude that $\nu^{t} \rightarrow \delta_{0}=\nu^{0}$ strongly in $M\left(\omega_{n}\right)$ and thus in $B(\omega)$ as $t \rightarrow 0$. 
We observe that condition (1) is only a slight strengthening of the standing assumption $(c): \sup _{t \in \mathbb{R}^{+}} \omega_{n+1}(t) / \omega_{n}(t)=\infty$ for every $n \in \mathbb{N}$; see the introduction. In order to show that (1) is a strictly stronger condition than (c), we construct below an unbounded weight $\omega$ which does not satisfy $\lim _{t \rightarrow \infty} \omega(t)=\infty$. We then let $\omega_{n}=\omega^{n}$ and observe that $\left(\omega_{n}\right)$ satisfies (c) but not 11 . The weight $\omega(t)=2^{v(t)}$ is constructed as follows. For $m \in \mathbb{N}$ let $v(m)$ be the number of 1's in the binary expansion of $m$ or equivalently the minimum number of powers of 2 needed to sum to $m$ and let $v(0)=0$. (This is a simplified version of the example given in [5, Example 9.17].) Then $v$ is subadditive and unbounded on $\mathbb{N}_{0}$, but does not tend to infinity as $m \rightarrow \infty$. The following lemma shows that the obvious extension of $v$ to $\mathbb{R}^{+}$stays subadditive. Hence $\omega$ is an algebra weight with the required properties.

LEMma 3.6. Let $v$ be a real-valued subadditive function on $\mathbb{N}_{0}$, and let also $v$ denote the continuous extension of $v$ to $\mathbb{R}^{+}$which is linear on each of the contiguous intervals. Then $v$ is subadditive on $\mathbb{R}^{+}$.

Proof. Let $x, y \in \mathbb{R}^{+}$with $x=m+r$ and $y=n+s$, where $m, n \in \mathbb{N}_{0}$ and $0 \leq r, s<1$. Observe that $v(x)=(1-r) v(m)+r v(m+1)$ and similar for $v(y)$. First, assume that $r+s \leq 1$. Then

$$
\begin{aligned}
v(x+y) & =(1-r-s) v(m+n)+(r+s) v(m+n+1) \\
& \leq(1-r-s)(v(m)+v(n))+s(v(m)+v(n+1))+r(v(m+1)+v(n)) \\
& =(1-r) v(m)+(1-s) v(n)+s v(n+1)+r v(m+1)=v(x)+v(y)
\end{aligned}
$$

as required. If $r+s>1$, we write $x+y=(m+n+1)+(r+s-1)$ and use the same approach as above.

For homomorphisms $\Phi: L^{1}\left(\omega_{1}\right) \rightarrow L^{1}\left(\omega_{2}\right)$ several conditions equivalent to the strong continuity of $\left(\nu^{t}\right)$ in $M\left(\omega_{2}\right)$ for $t \geq 0$ are given in [16, Corollary 3.13] and [14, Theorem 2.2]. The proofs of most of these equivalencies carry over directly (with obvious modifications) to endomorphisms of $A(\omega)$, so by using Theorem 3.5 , we obtain the result below. We say that $f \in A(\omega)$ is a standard element, if the closed ideal it generates in $A(\omega)$ is the standard ideal $A(\omega)_{d}=\{g \in A(\omega): \alpha(g) \geq d\}$, where $d=\alpha(f)=\inf \operatorname{supp}(f)$. Also, we say that an endomorphism $\Phi$ of $A(\omega)$ is a standard endomorphism, if whenever $f \in A(\omega)$ with $A(\omega) * f$ dense in $A(\omega)$, then $A(\omega) * \Phi(f)$ is dense in $A(\omega)$.

THEOREM 3.7. Let $\Phi$ be a non-zero endomorphism of $A(\omega)$, let $\left(\nu^{t}\right)$ be the semigroup from Theorem 3.2 and let $\left(e_{k}\right)$ be a bounded approximate identity for $A(\omega)$. Then the following are equivalent:

(a) $\left(\nu^{t}\right)$ is strongly continuous in $B(\omega)$ for $t \geq 0$.

(b) There is a non-zero standard element $f \in A(\omega)$ for which $\nu^{t} * f \rightarrow f$ in $A(\omega)$ as $t \rightarrow 0$.

(c) $\overline{\Phi(A(\omega))}$ contains a non-zero standard element.

(d) $\Phi$ is a standard endomorphism.

(e) $\left(\Phi\left(e_{k}\right)\right)$ is a bounded approximate identity for $A(\omega)$. 
(f) There is a non-zero standard element $f \in A(\omega)$ for which $\Phi\left(e_{k}\right) * f \rightarrow f$ in $A(\omega)$ as $k \rightarrow \infty$.

In particular, these properties all hold if condition (1) is satisfied.

Compared to the list in [14, Theorem 2.2] there are two notable omissions in Theorem 3.7. namely

(g) For every $h \in A(\omega)$ there exist $f, g \in A(\omega)$ such that $h=\Phi(f) * g$.

(h) The extension endomorphism $\widetilde{\Phi}$ of $B(\omega)$ is strongly continuous.

Considering $A(\omega)$ as a Fréchet $A(\omega)$-module under the action $f \cdot g=\Phi(f) * g$ for $f, g \in A(\omega)$, property (g) can be restated as existence of factorisation in this module, whereas (e) states that the sequence $\left(e_{k}\right)$ is a bounded approximate identity for this module. As mentioned in Section 2 we do not know whether factorisation in $A(\omega)$ and its modules follows from the existence of a bounded approximate identity, that is, whether (e) implies (g) (unless there exists $a>0$ such that $\sup _{n \in \mathbb{N}, 0 \leq t \leq a} \omega_{n}(t)<\infty$ ). However, the implications $(\mathrm{g}) \Rightarrow(\mathrm{h}) \Rightarrow(\mathrm{a})$-(f) follow rather easily as in the proof of [14, Theorem 2.2]. Moreover, it may be possible to prove (h) from (a) (without assuming (g)). For instance, it is easily seen that if $\left(\mu_{\beta}\right)$ is a bounded net in $B(\omega)$ with $\mu_{\beta} \rightarrow 0$ strongly, then $\widetilde{\Phi}\left(\mu_{\beta}\right) \rightarrow 0$ strongly in $B(\omega)$. We also mention in passing that if (a)-(f) hold, then $\widetilde{\Phi}$ is $\sigma$-continuous (see Section 2 for the definition): Let $\left(\mu_{\beta}\right)$ be a net in $B(\omega)$ with $\mu_{\beta} \rightarrow 0$ in the $\sigma$-topology, and let $n \in \mathbb{N}$. There exists $m \in \mathbb{N}$ such that $\widetilde{\Phi}$ extends to a continuous homomorphism $\widetilde{\Phi}_{n}: M\left(\omega_{m}\right) \rightarrow M\left(\omega_{n}\right)$. Since $\left(\nu^{t}\right)$ is continuous in $B(\omega)$ and thus in $M\left(\omega_{n}\right)$ for $t \geq 0$, it follows from [14, Theorem 2.2] that $\widetilde{\Phi}_{n}$ is strongly continuous. Since $\mu_{\beta} \rightarrow 0$ strongly in $M\left(\omega_{m}\right)$ we thus have $\widetilde{\Phi}\left({\underset{\sim}{\mu}}_{\beta}\right)=\widetilde{\Phi}_{n}\left(\mu_{\beta}\right) \rightarrow 0$ strongly in $M\left(\omega_{n}\right)$. Hence $\widetilde{\Phi}\left(\mu_{\beta}\right) \rightarrow 0$ in the $\sigma$-topology on $B(\omega)$, so $\widetilde{\Phi}$ is $\sigma$-continuous.

We end this section with a remark about automorphisms of $A(\omega)$. Characterisations of automorphisms have been obtained for the related algebras $L^{1}(\omega)$ ([8] and [12]), $L^{1}[0,1]$ $([20])$ and $L_{\text {loc }}^{1}\left(\mathbb{R}^{+}\right)([13])$. For the Fréchet algebra $L_{\text {loc }}^{1}\left(\mathbb{R}^{+}\right)$(which is the projective limit of the algebras $\left.L^{1}[0, n](n \in \mathbb{N})\right)$ the method consists of reducing the problem to automorphisms of $L^{1}[0,1]$. The following simple example indicates, that questions about automorphisms of $A(\omega)$ cannot easily be reduced to questions about isomorphisms between the algebras $L^{1}\left(\omega_{n}\right)(n \in \mathbb{N})$. Hence other methods are needed in order to obtain a characterisation of the automorphisms of $A(\omega)$. For $a \in \mathbb{R}^{+}$we define an algebra weight $\omega_{a}$ on $\mathbb{R}^{+}$by $\omega_{a}(t)=e^{a \sqrt{t}}$ for $t \in \mathbb{R}^{+}$. Moreover, for a function $f$ on $\mathbb{R}^{+}$we let $\Phi(f)(t)=2 f(2 t)$ for $t \in \mathbb{R}^{+}$. An easy calculation shows that $\|\Phi(f)\|_{\omega_{a}}=\|f\|_{\omega_{a / \sqrt{2}}}$ for $a \in \mathbb{R}^{+}$. With $\omega=\left(\omega_{n}\right)$ this shows that $\Phi$ is an automorphism of $A(\omega)$. However, for no $n \in \mathbb{N}$ is there an $m \in \mathbb{N}$ such that $\Phi$ extends to an isomorphism between $L^{1}\left(\omega_{m}\right)$ and $L^{1}\left(\omega_{n}\right)$ (but $\Phi$ does extend to an isomorphism between $L^{1}\left(\omega_{n / \sqrt{2}}\right)$ and $L^{1}\left(\omega_{n}\right)$ ).

4. Derivations. In Theorem 4.1 below we characterise the derivations on $A(\omega)$. Johnson ([19] or [4, Theorem 5.2.32]) proved that a semisimple, commutative Banach algebra does not have any non-zero derivations, and in particular this applies to each of the algebras $L^{1}\left(\omega_{n}\right)$. For (radical) weights $\omega$, Jewell and Sinclair (see [18, Remark 3(a)] or [4, Theorem 5.2.18 (ii)]) proved that derivations on $L^{1}(\omega)$ are automatically continuous. 
Moreover, Ghahramani ([8, Theorem 2.5]) showed that a linear operator $D$ on $L^{1}(\omega)$ is a derivation if and only if there is a measure $\mu$ on $\mathbb{R}^{+}$with

$$
\sup _{t \in \mathbb{R}^{+}} \frac{t}{\omega(t)} \int \omega(t+s) d|\mu|(s)<\infty
$$

such that $D(f)=(X f) * \mu$ for $f \in L^{1}(\omega)$, where $(X f)(t)=t f(t)$ for $t \in \mathbb{R}^{+}$and $f \in L^{1}(\omega)$.

Carpenter (1] or [15, Theorem 8.2.5]) partly generalised Johnson's result by showing that derivations on semisimple, unital Fréchet algebras automatically are continuous. We will see in Corollary 4.3 that this implies that derivations on $A(\omega)$ are continuous. (We remark that the proof of [4, Theorem 5.2.18 (ii)] (using a gliding hump technique) cannot be used in this case, since $A(\omega)$ is not locally bounded, that is, has no bounded open sets (as a ball in $L^{1}\left(\omega_{n}\right)$ is unbounded in $L^{1}\left(\omega_{m}\right)$ for $m>n$ ).)

Our main aim in this section is to prove the following result.

THEOREM 4.1.

(a) Suppose that

$$
\text { for every } n \in \mathbb{N} \text { there exists } m \in \mathbb{N} \text { such that } \sup _{t \in \mathbb{R}^{+}} \frac{t \omega_{n}(t)}{\omega_{m}(t)}<\infty .
$$

Then

$$
D_{\mu}(f)=(X f) * \mu \quad(f \in A(\omega))
$$

defines a continuous derivation on $A(\omega)$ for every $\mu \in B(\omega)$ and conversely every derivation on $A(\omega)$ has this form.

(b) If condition (2) is not satisfied, then there are no non-zero derivations on $A(\omega)$.

We remark that when condition (2) is satisfied, then $X$ (and thus $D_{\mu}$ ) extends to $B(\omega)$ by letting $d(X \nu)(t)=t d \nu(t)$ for $\nu \in B(\omega)$. Also, it is easily seem that condition (2) is equivalent with the formally weaker condition that there exists some $p>0$ such that for every $n \in \mathbb{N}$ there exists $m \in \mathbb{N}$ with

$$
\sup _{t \in \mathbb{R}^{+}} \frac{t^{p} \omega_{n}(t)}{\omega_{m}(t)}<\infty
$$

Theorem 4.1 and its proof are closely related to Ghahramani's result. Note however the difference in that we only have a condition on the weights $\left(\omega_{n}\right)$ and that if this condition is satisfied, then every $\mu \in B(\omega)$ gives rise to a derivation. We find it interesting that our results and methods for the "little" algebra $A(\omega)$ are similar to those used for the "big" algebras $M_{\text {loc }}\left(\mathbb{R}^{+}\right)([\underline{6}]), L_{\text {loc }}^{1}\left(\mathbb{R}^{+}\right)([13])$ and $L^{1}(\omega)$ for radical weights $\omega([\underline{8})$.

We need a few results in order to prove Theorem 4.1. Recall that the identification in Theorem 2.5 of $B(\omega)$ as the multiplier algebra of $A(\omega)$ induces a strong operator topology on $B(\omega)$.

Lemma 4.2. Every derivation $D$ on $A(\omega)$ extends to a derivation $\bar{D}$ on $B(\omega)$ which is norm- as well as strongly continuous.

Proof. We proceed as in [13, p. 57]: For $\mu \in B(\omega)$ we define a linear map $S_{\mu}$ on $A(\omega)$ by

$$
S_{\mu}(f)=D(\mu * f)-\mu * D(f) \quad(f \in A(\omega)) .
$$


It is easy to check that $S_{\mu}$ is a multiplier on $A(\omega)$. Hence it follows from Theorem 2.5 that there exists a unique measure $\bar{D}(\mu) \in B(\omega)$ such that $S_{\mu}(f)=\bar{D}(\mu) * f$ and thus

$$
D(\mu * f)=\bar{D}(\mu) * f+\mu * D(f) \quad \text { for } f \in A(\omega) .
$$

It is further easily seen that the map $\mu \mapsto \bar{D}(\mu)$ defines a derivation on $B(\omega)$ which extends $D$. Since $B(\omega)$ is semisimple by Corollary 2.4 it follows from Carpenter's result mentioned above that $\bar{D}$ automatically is continuous.

For the strong continuity of $\bar{D}$, let $\left(\mu_{\beta}\right)$ be a net in $B(\omega)$ with $\mu_{\beta} \rightarrow 0$ strongly. Then $\bar{D}\left(\mu_{\beta}\right) * f=D\left(\mu_{\beta} * f\right)-\mu_{\beta} * D(f) \rightarrow 0$ in $A(\omega)$ for every $f \in A(\omega)$, so $\bar{D}\left(\mu_{\beta}\right) \rightarrow 0$ strongly in $B(\omega)$ as required.

Corollary 4.3. Derivations on $A(\omega)$ are continuous.

The next result is similar to [6, Lemma 1]. Since the notation is somewhat different we include a proof for the sake of completeness. For a measure $\mu$ on $\mathbb{R}^{+}$we let $\alpha(\mu)=$ $\inf \operatorname{supp}(\mu)$.

Lemma 4.4. For a derivation $D$ on $B(\omega)$ we have

$$
\alpha(D(\mu)) \geq \alpha(\mu) \quad \text { for } \mu \in B(\omega) .
$$

In particular $\alpha\left(D\left(\delta_{t}\right)\right) \geq t$ for $t \in \mathbb{R}^{+}$.

Proof. Let $t \in \mathbb{R}^{+}$and $k \in \mathbb{N}$. Since $\delta_{t}=\left(\delta_{t / k}\right)^{* k}$ we have $D\left(\delta_{t}\right)=k\left(\delta_{t / k}\right)^{*(k-1)} * D\left(\delta_{t / k}\right)$, so it follows from Titchmarsh's convolution theorem that

$$
\alpha\left(D\left(\delta_{t}\right)\right) \geq \alpha\left(\left(\delta_{t / k}\right)^{*(k-1)}\right)=\frac{(k-1) t}{k} .
$$

Hence $\alpha\left(D\left(\delta_{t}\right)\right) \geq t$. Now let $\mu \in B(\omega)$ and let $t=\alpha(\mu)$. We may assume that $t>0$. Define the translate $\mu_{t} \in B(\omega)$ by $\mu_{t}(E)=\mu(E+t)$ for measurable sets $E \subseteq \mathbb{R}^{+}$. Then $\mu=\delta_{t} * \mu_{t}$, so $D(\mu)=D\left(\delta_{t}\right) * \mu_{t}+\delta_{t} * D\left(\mu_{t}\right)$, and thus

$$
\alpha(D(\mu)) \geq \min \left\{\alpha\left(D\left(\delta_{t}\right) * \mu_{t}\right), \alpha\left(\delta_{t} * D\left(\mu_{t}\right)\right)\right\} \geq \min \left\{\alpha\left(D\left(\delta_{t}\right)\right), \alpha\left(\delta_{t}\right)\right\}=t
$$

as required.

The proof of the following result is identical to that of [8, Lemma 2.3] (see also [6, Lemma 3]) and is therefore omitted.

Lemma 4.5. Let $D$ be a derivation on $B(\omega)$. Then there exists $\mu \in B(\omega)$ such that

$$
D\left(\delta_{t}\right)=t \cdot \delta_{t} * \mu \quad \text { for } t \in \mathbb{R}^{+} .
$$

The proof of Proposition 2.7 plays a crucial role in the proof of the next result.

Lemma 4.6. The linear span of $\left\{\delta_{t}: t \in \mathbb{R}^{+}\right\}$is strongly dense in $B(\omega)$.

Proof. Let $V$ be the linear span of $\left\{\delta_{t}: t \in \mathbb{R}^{+}\right\}$and let $\varphi$ be a strongly continuous linear functional on $B(\omega)$ with $\varphi\left(\delta_{t}\right)=0$ for $t \in \mathbb{R}^{+}$. From the proof of Proposition 2.7 it follows that $\varphi$ extends to a strongly continuous linear functional $\varphi_{N}: M\left(\omega_{N}\right) \rightarrow \mathbb{C}$ for some $N \in \mathbb{N}$. However, $V$ is strongly dense in $M\left(\omega_{N}\right)$ by [8, Lemma 1.3] (see also [6, Lemma 4]), so we deduce that $\varphi_{N}$ and thus $\varphi$ is zero, which finishes the proof. 
We are now ready to prove the characterisation of derivations on $A(\omega)$.

Proof of Theorem 4.1. (a): If condition (2) is satisfied, then $X$ defines a continuous linear operator $X: L^{1}\left(\omega_{m}\right) \rightarrow L^{1}\left(\omega_{n}\right)$ for every $n \in \mathbb{N}$ with corresponding $m \in \mathbb{N}$, and thus defines a continuous linear operator on $A(\omega)$. A routine calculation shows that $X$ is a derivation. Moreover, for $\mu \in B(\omega)$ the map $T_{\mu}(f)=\mu * f(f \in A(\omega))$ defines a continuous multiplier on $A(\omega)$ by Theorem 2.5. Hence $D_{\mu}=T_{\mu} X$ is a continuous derivation on $A(\omega)$.

Conversely, let $D$ be a derivation on $A(\omega)$ and let $\bar{D}$ be the continuous extension to a derivation on $B(\omega)$ given by Lemma 4.2. Also, let $\mu \in B(\omega)$ be the measure from Lemma 4.5 satisfying $\bar{D}\left(\delta_{t}\right)=t \cdot \delta_{t} * \mu$ for $t \in \mathbb{R}^{+}$. Define a continuous derivation $\Delta$ on $B(\omega)$ by

$$
\Delta(\nu)=\bar{D}(\nu)-(X \nu) * \mu \quad \text { for } \nu \in B(\omega) .
$$

Then $\Delta\left(\delta_{t}\right)=0$ for $t \in \mathbb{R}^{+}$. Furthermore, it follows from the proof of Lemma 4.2 that $\Delta$ is strongly continuous. Since the linear span of $\left\{\delta_{t}: t \in \mathbb{R}^{+}\right\}$is strongly dense in $B(\omega)$ by Lemma 4.6. we thus deduce that $\Delta=0$, so $\bar{D}(\nu)=(X \nu) * \mu$ for $\nu \in B(\omega)$ and thus $D=D_{\mu}$ as required.

(b): Assume that $D$ is a non-zero derivation on $A(\omega)$. As in the proof of (a), $D$ extends to a continuous derivation $\bar{D}$ on $B(\omega)$ and there exists $\mu \in B(\omega)$ such that $\bar{D}\left(\delta_{t}\right)=t \cdot \delta_{t} * \mu$ for $t \in \mathbb{R}^{+}$. Given $n \in \mathbb{N}$ it follows from Lemma 2.1 that there exists $m \in \mathbb{N}$ such that $D$ extends continuously to a map $D: L^{1}\left(\omega_{m}\right) \rightarrow L^{1}\left(\omega_{n}\right)$. Hence Grabiner's extension ([16. Theorem 3.4]) $\bar{D}: M\left(\omega_{m}\right) \rightarrow M\left(\omega_{n}\right)$ is also an extension of the derivation $\bar{D}$ on $B(\omega)$, so there exists a constant $c_{n}$ such that $\left\|\bar{D}\left(\delta_{t}\right)\right\|_{n} \leq c_{n}\left\|\delta_{t}\right\|_{m}$ for all $t \in \mathbb{R}^{+}$. However, $\left\|\bar{D}\left(\delta_{t}\right)\right\|_{n}=\int_{0}^{\infty} t \omega_{n}(s+t) d|\mu|(s) \geq t \omega_{n}(t)|\mu|\left(\mathbb{R}^{+}\right)$, whereas $\left\|\delta_{t}\right\|_{m}=\omega_{m}(t)$, so we conclude that (2) is satisfied.

5. Summary of open problems. We finish by gathering some open problems that have been mentioned in this paper.

(I) Do we have factorisation in $A(\omega)$ (and in Fréchet $A(\omega)$-modules) if $\lim _{n \rightarrow \infty} \omega_{n}(t)=$ $\infty$ for every $t \in \mathbb{R}^{+}$? (See the discussion preceding Theorem 2.5 )

(II) Are the $\sigma$-and the SO-topologies identical? (See the end of Section 2,)

(III) Are the conditions

(g) for every $h \in A(\omega)$ there exist $f, g \in A(\omega)$ such that $h=\Phi(f) * g$,

(h) the extension endomorphism $\widetilde{\Phi}$ of $B(\omega)$ is strongly continuous,

equivalent to the conditions in Theorem 3.7? (See the discussion following Theorem 3.7 )

Acknowledgements. This paper is based on a lecture delivered at the $19^{\text {th }}$ International Conference on Banach Algebras held at Będlewo, July 14-24, 2009. The support for the meeting by the Polish Academy of Sciences, the European Science Foundation under the ESF-EMS-ERCOM partnership, and the Faculty of Mathematics and Computer Science of the Adam Mickiewicz University at Poznań is gratefully acknowledged. 


\section{References}

[1] R. L. Carpenter, Continuity of derivations in F-algebras, Amer. J. Math. 93 (1978), 500502.

[2] J. B. Conway, A Course in Functional Analysis, Springer-Verlag, Berlin, 1985.

[3] I. G. Craw, Factorisation in Fréchet algebras, J. London Math. Soc. 44 (1969), 607-611.

[4] H. G. Dales, Banach Algebras and Automatic Continuity, London Mathematical Society Monographs, New Series 24, Oxford University Press, Oxford, 2000.

[5] H. G. Dales and A. T.-M. Lau, The second duals of Beurling algebras, Mem. Amer. Math. Soc. 177 (2005).

[6] H. G. Diamond, Characterization of derivations on an algebra of measures, Math. Z. 100 (1967), 135-140.

[7] R. S. Doran and J. Wichmann. Approximate Identities and Factorization in Banach Modules, Lecture Notes in Math. 768, Springer-Verlag, Berlin, 1979.

[8] F. Ghahramani, Homomorphisms and derivations on weighted convolution algebras, J. London Math. Soc. (2) 21 (1980), 149-161.

[9] F. Ghahramani, Endomorphisms of $L^{1}\left(\mathbb{R}^{+}\right)$, J. Math. Anal. Appl. 85 (1982), 308-314.

[10] F. Ghahramani and S. Grabiner, Standard homomorphisms and convergent sequences in weighted convolution algebras, Illinois J. Math. 36 (1992), 505-527.

[11] F. Ghahramani and S. Grabiner, Convergence factors and compactness in weighted convolution algebras, Can. J. Math. 54 (2002), 303-323.

[12] F. Ghahramani and J. P. McClure, Automorphisms of radical weighted convolution algebras, J. London Math. Soc. (2) 41 (1988), 122-132.

[13] F. Ghahramani and J. P. McClure, Automorphisms and derivations of a Fréchet algebra of locally integrable functions, Studia Math. 103 (1992), 51-69.

[14] F. Ghahramani, J. P. McClure, and S. Grabiner, Standard homomorphisms and regulated weights on weighted convolution algebras, J. Funct. Anal. 91 (1990), 278-286.

[15] H. Goldmann, Uniform Fréchet Algebras, North-Holland Mathematics Studies 162, NorthHolland, Amsterdam, 1990.

[16] S. Grabiner, Homomorphisms and semigroups in weighted convolution algebras, Indiana Univ. Math. J. 37 (1988), 589-615.

[17] S. Grabiner, Weak ${ }^{*}$ properties of weighted convolution algebras II, Studia Math. 198 (2010), 53-67.

[18] N. P. Jewell and A. M. Sinclair, Endomorphisms and derivation on $L^{1}(0,1)$ are continuous, Bull. London Math. Soc. 8 (1976), 135-139.

[19] B. E. Johnson, Continuity of derivations on commutative algebras, Amer. J. Math. 91 (1969), 1-10.

[20] H. Kamowitz and S. Scheinberg, Derivations and automorphisms of $L^{1}(0,1)$, Trans. Amer. Math. Soc. 135 (1969), 415-427.

[21] E. A. Michael, Locally multiplicatively-convex topological algebras, Mem. Amer. Math. Soc. 11 (1952).

[22] T. V. Pedersen, Weak-star properties of homomorphisms from weighted convolution algebras on the half-line, J. Austral. Math. Soc. 89 (2010), 75-90.

[23] W. Rudin, Functional Analysis, McGraw-Hill, New York, 1973.

[24] M. K. Summers, Factorization in Fréchet modules, J. London Math. Soc. (2) 5 (1972), 243-248. 
